\title{
NOTICES INDUSTRIELLES
}

\author{
Université de Grenoble. - Institut Polytechnique.
}

Grenoble, le 15 février 1922.

Monsieur et cher Confrère,

Permettez-moi, au début de cette année, d'attirer encore votre bienveillante attention sur un certain nombre d'élèves sortants de l'Institut Electrotechnique, appartenant tous à nos promotions spéciales, dites militaires, et dont la dernière, composée de 125 ingénieurs, tous très intéressants, autant par leur valeur morale et technique, que par leur passé militaire, vient de quitter notre Etablissement.

Vous savez quel effort considérable nous avons fourni, mes collègues et moi, ayant tous été du reste mobilisés pendant la guerre, en faveur de nos jeunes camarades. Ces efforts ont été couronnés de succès, puisque nos trois premières promotions militaires spéciales se sont toutes placées sans grande difficulté. II s'agit d'un dernier effort à tenter en faveur de jeunes gens remarquablement méritants.

Le grand nombre de nos élèves, bien que cet afflux ait été dû à la présence des seuls démobilisés, a éveillé quelques susceptibilités ou provoqué quelques craintes. Vous nous connaissez assez pour savoir que nous n'avons pas voulu à Grenoble, faire du nombre, mais simplement faciliter les études techniques à des jeunes gens qui les avaient commencées avant la guerre, ou tout au moins qui se destinaient à l'industrie et que l'extrême longueur du cycle d'études correspondant à la préparation et au travail des Grandes Ecoles avait quelque peu effrayés.

Peut-on reprocher à des étudiants de 25 ans d'avoir redouté de ne pas être à même de gagner leur vie avant trente ans !

Cette dernière promotion épuisée, notre fonctionnement à l'Institut redeviendra normal. Nous vous demandons done instamment de nous aider à favoriser les débuts dans la cárrière de ces jeunes gens, presque tous décorés de la croix de guerre, beaucoup même possesseurs de la Légion d'Honneur ou de la Médaille Militaire.

Dans l'espoir que vous voudrez bien nous aider dans cette œuvre de justice sociale, nous vous prions d'agréer, Monsieur et cher Confrère, nos meilleurs et bien dévoués sentiments.

L. BARBILlion, Professeur d la Faculté des sciences, Directeur de l'Institut Electrotechnique.

\section{Le Ministre du Travail visite les Usines de la C. A. M. (Roulements à billes R. E. F.)}

M. Albert Peyronnet, ministre du travail, accompagné de M. Debierre, senateur du Nord, a visité, vendredi 3 courant, les importantes usines de la Compagnie d'Applications Mécaniques (roulements à billes R. B. F.), à Ivry-Port. Il a été reçu par M. Théodore Hoffmann, administrateur directeur général de la Compagnie.

Le ministre s'est plus particulièrement intéressé aux œuvres sociales (infirmerie, salle d'opérations, œuvres d'assistance féminine, primes à la maternité, à l'allaitement, sursalaires familiaux) ainsi qu'aux méthodes de travail modernes adaptées d'une façon aussi souple que complète aux diverses opérations de fabrication, par l'Administrateur Directeur général de la C. A. M. , qui sut obtenir dans ce domaine des résultats remarquables quì placent cette Compagnie au premier rang des industries similaires.
Les services de contrôle et de vêfification de la fabrication, ainsi que les laboratoires d'essais et de recherches mécaniques, physiques et chimiques retinrent très longuement l'attention du ministre du travail, car, comme on le sait, l'industrie du roulement à billes exige des contrôles permanents des matières premières et une grande précision d'usinage. Il fut très intéressé par les diverses expériences exécutées sous ses yeux et félicita tout particulierement M. Théodore Hoffmann de l'effort accompli.

Quant à l'hygiène des ateliers, il fit remarquer aux personnes qui l'accompagnaient la propreté des divers locaux et leur bonne condition de salubrité et de confort.

Le Ministre quitta lés usines, accompagné de MM. Debierre et Diquenoy, directeur de l'expansion à la Compagnie.

\section{Demande de Pompes en Égypte.}

L'Egypte essentiellement agricole et où il ne pleut que rarement, utilise beaucoup de pompes pour l'arrosage de ses nombreuses cultures.

Il n'y a pas de types de pompes spécialement préférés, les principaux types sont employés également.

On utilise les pompes centrifuges montées sur des machines à vapeur ou des moteurs à pétrole lourd, pétrole lampant ou à benzine, de toutes dimensions et de tous diamètres de tubulures. Ces pompes sont employées pour les puits artésiens.

On utilise également les pompes rotatives et semi-rotatives et les pompes à piston. Les pompes rotatives à main et les petites pompes à piston sont surtout utilisées pour l'alimentation en eau de gros mateurs, ou machines à vapeur des grosses pompes. On les utilise aussi pour les usages domestiques dans les villages.

On emploíe beaucoup en Egypte une petite pompe à main ou à moteur qu'on appelle pompe abyssinienne et qui vient principalement d'Amérique, de provenance allemande avant la guerre.

La pompe alimentaire appelée aussi pompe petit cheval, qui sert à l'alimentation de gros moteurs à refroidissement à, eau, vient également d'Amérique.

L'Amérique fait en ce moment une très grosse concurrence sur ce genre d'article qui venait avant la guerre d'Angleterre, de Suisse, d'Allemagne, de France.

Droits de douane : $81 / 2 \%$ ad valorem.

\section{Arrêté approuvant le Compteur électrique type L. C.}

D'après le Journal Officiel du 22 février, page 2235, par arrêté du ministre des Travaux publics en date du 20 février 1922, pris aprés avis du Comité d'Electricité, a été approuvé, en conformité de l'article 16 des cahiers des charges des concessions de distribu- tion d'énergíe électrique, le compteur ampère-heure mètre à courant continu type $L$. C. pour les installations à deux fils et jusqu'a dix ampères, présenté par le Sangamo Electric Company, dont le siege social est à Springfield (minois). 


\section{INFORMATIONS}

\section{Au sujet de l'Aménagement du Rhône.}

Au cours de la deuxième réunion de la conférence préparatoire pour la constitution de la Compagnie Nationale du Rhône, qui s'est tenue hier matin à l'Hôtel de Ville, toutes les questions ont pu être envisagées. Comme nous l'avions laissé prévoir, un accord complet s'est fait sur toutes les questions, et notamment sur le programme des travaux et l'ordre d'urgence de leur exécution. Tous les travaux relatifs aux forces hydrauliques, à la navigation et à l'irrigation seront menés parallèlement.

On s'est également entendu sur l'ordre d'exécution des travaux. La première tranche comprendra l'aménagement des chutes des sections de Génissiat et de Donzère-Mondragon, ainsi que celui du port de Lyon et des divers ports à l'aval de cette ville. On comprendra également dans la première partie des travaux la construction des deux "feeders "Génissiat-Paris et Arles-Génissiat.

Sur la question d'irrigation, qui avait donné lieu à un assez vif incident, on a encore abouti à un accord parfait. Il a été entendu qu'à toute époque de la concession, les intéressés pourraient réclamer l'exécution des canaux primaires et des stations de pompage. Lorsque des associations reconnues par le Ministère de l'Agriculture auront été constituées, le tarif de pompage sera d'un prix réduit.

Le gouvernement français a déclaré qu'il inviterait le gouver- nement suisse à pousser très activement les pourparlers relatifs à l'utilisation maximum de la capacité d'emmagasinement du lac Léman.

La première tranche des travaux est évaluée, approximativement, à 1.150 millions et la totalité des travaux à 3 milliards 300 millions.

L'accord étant fait sur toutes les questions, les représentants des départements procéderont aux consultations nécessaires à la détermination du capital social. Les Conseils généraux traiteront vraisemblablement ce problème au cours de la session d'avril.

Aussitôt après, la section permanente de la Commission interdépartementale se réunira et examinera l'ensemble de ces délibé:rations.

Il est probable qu'une réunion générale pourra se tenir dans le courant du mois de juin et sera alors dans la possibilité de mettre au point les statuts de la Compagnie Nationale du Rhône.

La réunion d'hier s'est donc terminée sous une impression de confiance et d'optimisme. M. Léon Perrier a adjuré les intéressés de faire abstraction de leurs intérêts personnels et de ne considérer que les intérêts généraux, de former une sorte d'union sacrée pour la réalisation de cette immense entreprise, quí aura dans la nation française une si utile répercussion.

\section{Le grand Canal d'Alsace et l'Aménagement du Rhin entre Bâle et 'Strasbourg.}

La réalisation rapide de l'aménagement du Rhin entre Bâle et Strasbourg préoccupe à bon droit l'opinion publique suisse qui désirerait que la ville de Bâle soit raccordée le plus tôt possible à la mer par une voie navigable permettant un trafic important.

Certains ont reproché à ce sujet à l'administration française de présenter pour cet aménagement un projet trop grandiose le projet du grand canal d'Alsace - et ont peur que l'exécution de ce travail dure de longues années.

Il faut remarquer cependant que la France pousse très activement les études techniques et financières relatives à son projet : elle a besoin de la force motrice du Rhin et a tout intérêt à réaliser rapidement la grande artère navigable prévue entre Strasbourg, Mulhouse, Lyon et Marseille.

Supposons cependant que cette réalisation soit un peu plus longue qu'on ne l'espère. La France s'opposerait-elle à toute amélioration provisoire du chenal navigable du Rhin.dans les parties où le canal ne serait pas construit?

Pas le moins du monde et la déclaration suivante qui a été faite par la délégation française au cours de la dernière session de la Commission Centrale pour la Navigation du Rhin en fait foi :

"Pendant la période d'exécution du canal latéral entier, si un gouvernement, que l'amélioration de la navigation intéresse, prend l'initiative de proposer l'exécution de certains travaux d'améliora- tion dans les sections du lit auxquelles le canal latéral n'aura pas encore été substitué, et si la Commission Centrale donne son approbation à ces travaux, la France accepte d'examiner avec ses coriverains les mesures propres à en faciliter la réalısation, le tout sous réserve qu'il reste bien entendu que les sections améliorées seront remplacées par le canal au fur et à mesure que les sections correspondantes du canal seront admises en exploitation. "

Cette déclaration, qui fait preuve du plus large esprit de conciliation, montre que la France est toute disposée à faciliter la navigation en amont de Strashourg. Elle a d'ailleưrs un grand intérêt à ce que la région industrielle de Mulhouse et le bassin de potasse qui avoisine cette ville soient accessibles le plus tôt possible à la navigation rhénane.

Il n'y a donc pas d'oppositions entre les aspirations suisses et françaises, comme ont essayé de le montrer vainement lès adversaires du canal d'Alsace, mais il existe, au contraire, entre les deux pays, une véritable communauté d'intéréts dans la question rhénane. Cette communauté d'intérêts est un sûr gäant que les besoins de la navigation ne seront pas négligés et que les vœux les plus chers de la Suisse en ce qui concerne le Rhin supérieur seront promptement réalisés si tous les intéressés se mettent d'accord comme ils le doivent pour travailler en commun.

(Agence Rhin)

\section{Chambre de Commerce de Grenoble. - XII ${ }^{\mathrm{e}}$ Groupement économique régional de Chambres de Commerce.}

\section{COMTTÉ D'ADMINISTRATION}

Réunion tenue à Nice le 28 janvier 1922.

Le Comité régional d'Administration de la XIIe région économique a tenu sa première réunion annuelle à Nice, le samedi 28 janvier écoulé.

La séance commencée le matin, à 9 heures $3 / 4$, interrompue à midi par un déjeuner officlel offert par la Chambre de Commerce de Nice à tous les membres du Comité, reprise à 15 heures, s'est terminée le soir à 18 heures.

Le Comité, après avoir approuvé les comptes de gestion de l'année 1921 et adopté le projet de budget établi pour l'année 1923, prend les décisions suivantes : $1^{\circ}$ Il décide de réunir la Commission plénière prévue au prògramme du concours institué pour la construction de la ligne directe Grenoble-Nice, vers la fin du mois de juin 1922.

Le Comité spécial d'ingénieurs qui avait été chargé d'examiner et de classer les projets déposés en vue de ce concours a, en effet, terminé ses travaux, et son rapport définitif sera lu et discuté au cours de la réunion dont il s'agit. Cette réunion enfin aura lieu à Grenoble et la plus grande publicité sera donnée aux opérations du concours.

$2^{\circ}$ Il décide d'entreprendre une enquête sur la Route des Alpes. Cette enquête, qui sera faite sur place et en pleine harmonie avec les Syndicats d'initiative, les Syndicats d'hôteliers et de transporteurs, la Compagnie P.-L.-M., les services des Ponts et Chaus- 
sées et des Eaux et Forêts, des P. T. T., etc..., aura pour but de rechercher les améliorations dont la grande artère alpine d'été serait susceptible : bon état de la route, rectification de certains virages, croisements, pose de poteaux indicateurs, plantations d'arbres pour donner de l'ombrage, communications téléphoniques, organisations de secours rapide en cas d'accidents, jonction convenable des secteurs, hôtels à construire, etc., etc...

$3^{0}$ Il décide de poursuivre la réalisation de son projet de construction d'un certain nombre d'hôtels de montagne dont le principe est admis à l'unanimité. Il décide, à cet effet, de rechercher de suite les points de la Route des Alpes sur lesquels il conviendrait soit de construire un hôtel neuf, soit d'aménager confortablement un' hôtel déjà existant après l'avoir acheté

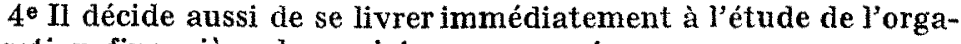
nisation financière du projet comprenant :

a) La fixation de l'emprunt total à contracter ;

b) Le partage de cet emprunt entre les Chambres de[Commerce participantes ;

c) La répartition des dépenses à engager sur chaque point de la région des Alpes désigné pour recevoir un hôtel neuf ou transformé ;

$5^{\mathrm{e}}$ Il émet un vœu favorable à la construction du tronçon de ligne Briançon-Oulx, sous la réserve que cette construction ne gênera en rien les autres projets à l'étude destinés à relier les réseaux ferrés français au réseau italien.

\section{A l'Union des Syndicats de l'Electricité.}

L'Union des Syndicats de l'Electricité vient de tenir son Assemblée générale et a reçu à cette occasion communication du rapport sur les travaux de l'exercice écoulé.

Ce rapport qui montre en détail le rôle qu'a joué l'Union au triple point de vue des études juridiques, économiqués et techniques, est malheureusement trop long pour que nous puissions le reproduire.

Chacun connaît d'ailleurs l'importance des travaux de normalisation qui sont poursuivis depuis plusieurs années par l'U. S. E. et qui ont été utilisés non seulement en France, notamment par la Commission permanente de Standardisation, mais aussi à l'étranger.

Le rapport s'est étendu sur la Conférence Internationale des
Grands Réseaux Electriques à Très Haute Tension, que l'U.S.E. avait organisée au mois de novembre dernier et dont le succès a été si marqué que de nouvelles sessions vont avoir lieu régulièıement tous les deux ans à Paris.

Deux nouveanx syndicats viennent de se faire inscrire à l'U. S. E. : la section des tramways de l'Union des voies ferrées d'Intérêt local et le Syndicat des Entrepreneurs de Grands Réseaux et de Centrales Electriques, ce dernier de constitution toute récente.

Le rapport indique enfin que, par suite de l'extension des services de l'U. S. E., le siège social va être transféré incessamment au No 25 du boulevard Malesherbes (Tél. : Elysées 31-82).

\section{Aménagement d'une Chute de l'Ain.}

Par décret du 6 juin 1918 ont été autorisés et déclarés d'utilité publique les travaux de dérivation de l'Ain, entre Pont d'Ain et Priay, pour la mise en jeu d'une usine hydroélectrique ; par un décret ultérieur, du 11 mai 1920, ont été autorisés et déclarés d'utilité publique les travaux d'aménagement de la chute de l'Ain entre Priay et Gévrien.

Les concessionnaires, MM. Vuillermoz et Tezenas, ayant demandé une, prolongation de deux ans des délais relatifs à ces con- cessions, un décret du ministre des Travaux publics en date du 20 février 1922, publié au Journal Officiel du 23 février, page 2257, vient de proroger jusqu'au 11 mai 1923 le terme du délai fixé pour la constitution d'une société anonyme et de la présentation des projets d'exécution et jusqu'au 11 mai 1924 le terme du délai fixé pour l'expropriatir a des terrains nécessaires à l'exécution des ouvrages.

\section{Construction d'une Usine hydro-électrique dans l'Isère.}

Par décret du ministre des Travaux publics en date du 18 février 1922, publié au Journal Officiel du 23 février, page 2257, est approuvée la substitution de la Société Energie électrique Isère-Vercors, dont le président est M. Boissonnas, à la Société anonyme des Forces motrices du Vercors dans les droits et obli- gations de celle-ci résultant du décret du 12 janvier 1921, approuvant la convention passée entre le ministre des Travaux publics et cette dernière société potur la construction d'un barrage sur l'Isère, au quartier de Pizançon. et la construction d'une usine hydroélectrique.

\section{Société d'Électrochimie et d'Électrométallurgie.}

Les actionnaíres de cette Société, dont le siège est à Paris, 2, rue Blanche, réunis dernièrement en assemblée extraordinaire, après avoir pris connaissance de l'acte d'apport-fusion en date du 17 janvier 1922, intervenu avec la Compagnie des Forges et Aciéries électriques Paul Girod, ont approuvé, en principe, le projet de fusion de cette société, au moyen de son absorption par la Société d'Electrochimie et d'Electrométallurgie dans les conditions déterminées par l'acte précité.

En conséquence, et sous réserve de la réalisation définitive de la fusion envisagée, l'assemblée a autorisé la création de 36.000 actions nouvelles entièrement libérées de la Société d'Electrochimie et d'Electrométallurgie, nécessaires pour permettre l'attribution prévue en faveur de la Compagnie des Forges et Aciéries électriques Paul Girod, soit trois actions Electrochimie pour cinq actions Paul Girod.

D'autre part, l'Assemblée, après avoir pris connaissance des accords intervenus avec la Compagnie nationale des Matières colorantes et de Produits chimiques, aux termes desquels elle `’est engagée à faire apport à la Société d'Electrochimie et d'Electrométallurgie de son usine de Jarrie-Vizille et dépendance, a approuvé, en principe, ces accords.
En conséquence, et sous réserve de la réalisation définitive de l'apport, l'Assemblée a autorisé la création de 2.900 actions nouvelles de 500 francs, entièrement libérées, de la Société d'Electrochimie et d'Electrométallurgie, à attribuer à titre de prix partiel dudit apport, le solde du prix de cet apport consistant en un versement de 2.500.000 fr. en espèces, à effectuer.

Par suite des décisions ci-dessus, le capital social se trouve porté à $36.450 .000 \mathrm{fr}$., et divisé en 72.900 actions de $500 \mathrm{fr}$.

Sous condition suspensive de la réalisation définitive de la fusion projetée, les articles $2,5,16,21,28,31,32$ et 40 des statuts ont été modifiés.

L'Assemblée générale a décidé que lorsque l'un ou l'autre des deux apports ci-dessus prévus aura été délinitivcment réalisé, le Conseil d'Administration aura tous pouvoirs pour augmenter, en une ou plusieurs fois, le capital en vue de le porter à 80 millions de francs par l'émission d'actions nouvelles à souscrire contre espèces.

Le Conseil d'Administration est autorisé à procéder à cette ou ces augmentations de capital s'il le juge convenable et quand il l'estimera opportun, et à fixer en conséquence toutes lés conditions y afférentes. 


\section{Compagnie Française d'Installations électriques.}

D'après une insertion au Bulletin des Annonces légales et obligatoires du 20 février 1922, page 96, cette société en formation, dont le siège est à Thouars, a pour objet : tous travaux se rapportant aux distributions d'énergie électrique ; l'achat, la vente, la fabri- cation, la réparation, la location de tous appareils se rapportant aux applications de l'électricité et à son utilisation.

Le capital est fixé à 1 million de francs, divisé en 4.000 actions de $250 \mathrm{fr}$.

\section{Omnium Français d'Électricité.}

D'après une insertion au Bulletin des Annonces légales et Obligatoires du 27 février 1922 , p. 113 , cette société, dont le siège est à Paris, 157, boulevard Péreire, va procéder à l'émission de 4.000 obli- gations de $500 \mathrm{fr}$. chacune, rapportant un intérêt annuel de 7 poưr 100 , net d'impôts présents et futurs, remboursables au pàir en trente-sept ans. Ces obligations sont émises à 495 fr.

\section{L'Électrique de Bretagne.}

D'après une insertion au Bulletin des Annonces légales et obligatoires du 20 février 1922, p. 97, cette société, dont le siège est à Rennes, 24, boulevard de la Liberté, va procéder à l'émission de 2.000 obligations de $500 \mathrm{fr}$. chacune, rapportant un intérêt annuel de 7 pour 100, net de tous impôts présents et futurs, et remboursables en 40 ans à partír du 1ẹr juillet 1925. Ces obligations sont émises à $495 \mathrm{fr}$.

\section{Compagnie générale électrique de Nancy et Société des Fonderies, Laminoirs et Tréfileries de Vitry-sur-Seine.}

L'assemblée extraordinaire qui a eu lieu dernièrement a décidé de porter le capital de cette société de 2,5 millions à 10 millions de francs. C'est la conséquence' d'un accord conclu entre la Compagnie générale électrique de Nancy qui détient toutes les actions

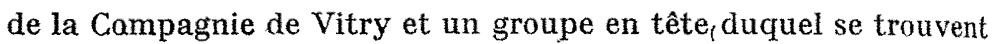
Dives et la Compagnie générale d'Electricité qui a décidé de s'intéresser à cette très importante affaire de laminage de cuivre en y prenant une participation de 50 pour 100 .

\section{Ateliers de Constructions électriques de Lyon et du Dauphiné.}

Réunis en assemblée extraordinaire à Lyon, le 18 février 1922, les actionnaires de cette Société ont approuvé à l'unanimité toutes les résolutions présentées.

Elle a notamment approuvé et accepté provisoirement l'apport par voie de fusion effectuée par la Société des Etablissement, Maljournal et Bourron, entraînant ainsi une augmentation de capital par la création d'actions à attribuer en rémunération d'apports.

\section{Énergie électrique Isère-Vercors.}

La substitution de cette société à la Société anonyme des Forces Motrices du Vercors, pour l'établissement d'un barrage súr l'Isère

et la mise en jeu d'une usine hydroélectrique a été autorisée par décret en date du 18 février 1922.

\section{Forces motrices de la Tardes.}

Cette société, qui a pour objet l'établissement et l'exploitation d'usines électriques centrales, vient de transférer son siège de Chambon-sur-Voueize à Chénerailles (Creuse).

\section{Forces motrices}

L'assemblée ordinaire du 18 février a autorisé cette société à passer avec une société fermière au capital de un million de frąncs une convention pour l'exploitation en régie de ses usines hydroélec-
La société possède un barrage sur la Tardes, un peu en aval de Mazeirat, commune de Tardes (Creuse).

\section{de la Garonne.}

triques et électro-chimiques de Mancioux et de Boussens (HauteGaronne).

\section{Compagnie régionale de Distribution d'Électricité.}

Une assemblée extraordinaire des actionnaires de cette société, dont le siège est à Paris, 10 , place de la Bourse, a eu lieu le 22 février. Elle a décidé l'émission de 20.000 actions de $100 \mathrm{fr}$., afin de porter le capital social de 154.000 fr. à 2.154 .000 fr.
Le Conseil a, en outre, reçu toutes autorisations nécessaires pour effectuer un emprunt obligataire de $1.200 .000 \mathrm{fr}$. en sus d'un emprunt obligataire de $500.000 \mathrm{fr}$. autorisé par les statuts.

\section{Société régionale de Distribution d'Électricité.}

Cette société, dont le siège est à Paris, 131, boulevard Péreire, a tenu le 12.février une assemblée extraordinaire au cours de laquelle elle a décidé de réduire son capital de $600.000 \mathrm{fr}$. à $300.000 \mathrm{fr}$. par l'abaissement à $250 \mathrm{fr}$. du nominal actuel de $500 \mathrm{fr}$. des actions.
D'autre part, elle a décidé de porter le capital ainsi réduit à $400.000 \mathrm{fr}$. par l'émission de 1.000 actions privilégiées de $100 \mathrm{fr}$. 7 pour 100 net d'impôts.

\section{Société d'Électricité de la Vallée du Rhône.}

Cette société, dont le siège est à Lyon, 4, rue Martin, va porter son capital de 1.800 .000 fr. à 3.000 .000 fr. par l'émission de 2.400 actions nouvelles de $500 \mathrm{fr}$. payables comme suit : un quart à la souscription ef le surplus en une ou plusieurs fois aux dates qui seront déterminées par le Conseil d'administration.

Les nouvelles actions sont réservées par préférence aux anciens actionnaires à raison de deux actions nouvelles pour trois anciennes, sur présentation du titre ancien. 